Research Article

\title{
Worst-Case Performance Optimization Beamformer with Embedded Array's Active Pattern
}

\author{
Yuyue Luo $\mathbb{D}^{1}{ }^{1}$ Jin Pan, ${ }^{1}$ J. Andrew Zhang, ${ }^{2}$ and Shaode Huang $\mathbb{D}^{1}$ \\ ${ }^{1}$ School of Electronic Science and Engineering, University of Electronic Science and Technology of China, Chengdu 611731, China \\ ${ }^{2}$ University of Technology Sydney, Ultimo, NSW, Australia \\ Correspondence should be addressed to Yuyue Luo; yyl_uestc@163.com
}

Received 15 December 2017; Revised 13 March 2018; Accepted 22 April 2018; Published 24 June 2018

Academic Editor: Francesco D'Agostino

Copyright (c) 2018 Yuyue Luo et al. This is an open access article distributed under the Creative Commons Attribution License, which permits unrestricted use, distribution, and reproduction in any medium, provided the original work is properly cited.

\begin{abstract}
This paper proposes an adaptive array beamforming method by embedding antennas' active pattern in the worst-case performance optimization algorithm. This method can significantly reduce the beamformer's performance degradation caused by inconsistency between hypothesized ideal array models and practical ones. Simulation and measured results consistently demonstrate the robustness and effectiveness of the proposed method in dealing with array manifold mismatches.
\end{abstract}

\section{Introduction}

The assumption of ideal array elements in conventional adaptive beamforming technologies can cause severe performance degradation in real implementations due to ignored array imperfections (e.g., gain and phase mismatches and mutual coupling between elements), particularly for increasingly widely used small-profile arrays. Robust beamforming algorithms have been proposed to deal with these imperfections by treating an array's response inconsistencies as nonspecific manifold mismatches. There are some classic algorithms, such as the diagonal loading (DL) method (also called loaded sample matrix inversion (LSMI) beamformer) [1] and the worst-case performance optimization robust beamformer (WCRB) [2], summarized in [3, 4]. The WCRB approach [2] is also extended and applied to several specific scenarios [5-8]. However, most robust beamforming methods solve uncertain problems based on simplified array models, without considering the array's electromagnetic characteristics, which are actually essential to the manifold mismatches and are critical for the performance of the methods in practice.

The problem of array modelling mismatches is typically studied by antenna researchers. An earlier work exploiting the gain and frequency properties of practical antennas was reported in [9], without considering mutual coupling effect. In [10], improvement to [9] is made by incorporating the antenna's active pattern (AP) introduced in [11], which calculates an elements' radiation and its impact on the array environment (both mutual coupling between elements and workspace radiation) [12]. However, these methods rely on the exact knowledge of antennas' electromagnetic characteristics and are quite sensitive to measurement mismatches.

In this paper, by creatively integrating antenna mismatch modelling into beamforming design, we propose a robust worst-case performance optimization beamformer with an embedded array's AP. We call it as active pattern worst-case (APWC) method which can significantly improve the beamformer robustness under various mismatches. The APWC method essentially introduces the AP method [10] into the WCRB algorithm [2]. Via both simulation and experiments with real measurements, we demonstrate that the APWC beamformer can achieve significantly better performance (e.g., higher signal-to-interference-and-noise ratio (SINR)) than many existing schemes. It has better tolerance to both engineering and electromagnetic mismatches caused by elements' modelling, manufacturing, aperture assembling, and channel debugging. 


\section{Problem Formulation}

We consider an $M$-element two-dimension antenna array and a narrowband system. Without considering any imperfections, its steering vector $\mathbf{a}_{s}$ can be represented as

$$
\mathbf{a}(f, \theta, \varphi, \mathbf{r})=\left[1, e^{j k \mathbf{r}_{1}(\theta, \varphi)}, \ldots, e^{j k \mathbf{r}_{M-1}(\theta, \varphi)}\right],
$$

where $k=2 \pi f / v$ is the wavenumber; $f$ and $v$ denote the frequency and the speed of the electromagnetic wave, respectively; $\theta$ and $\varphi$ are the angles; and $\mathbf{r}_{i}(\theta, \varphi)$ is the $(i+1)$ th sensor's location vector.

Assume omnidirectional antenna elements. Let $s(t)$ be the transmitted data symbol at time $t$. The signal received at the array is given by

$$
\mathbf{x}(t)=s(t) \mathbf{a}_{s}+i(t) \mathbf{a}_{i}+\mathbf{n}(t)
$$

where $\mathbf{x}(t)=\left[x_{1}(t), x_{2}(t), \ldots, x_{M}(t)\right]^{T} \in C^{M \times 1}, \mathbf{a}_{s}$, and $\mathbf{a}_{i}$ are the steering vectors of signal and multiuser interferences, respectively, and $\mathbf{n}(t)$ is an $M \times 1$ vector denoting the combined self-interference from mismatches and noise components.

The well-known sample matrix inversion (SMI) beamformer solves a constrained minimization problem

$$
\begin{array}{ll}
\min _{\mathbf{w}} & \mathbf{w}^{H} \widehat{\mathbf{R}} \mathbf{w}, \\
\text { s.t. } & \mathbf{w}^{H} \mathbf{a}=1,
\end{array}
$$

where $(\cdot)^{\mathrm{H}}$ denotes Hermitian transpose, $\mathbf{w}$ is the complex beamforming vector, and $\widehat{\mathbf{R}}$ is the sample covariance matrix of $\mathbf{x}(t)$.

\section{Improved Model for Array Steering Vector}

There are always mismatches, that is, the fluctuation of array parameters during design, processing, measuring, and assembling, between the ideal steering vector $\mathbf{a}$ and the actual one $\mathbf{a}$. Mismatches generally have a minor impact on the electromagnetic characteristics of a single antenna element, such as current distribution and boundary conditions and, hence, cause small changes to each element's basic radiation structure, as well as the radiation pattern and directivity. Such small changes, however, when beamforming is formed, can cause large beamforming gain variations, due to gain, sometimes phase, misalignment between different antenna elements [13]. In practice, an array's radiation performance can be significantly affected by such array mismatches.

In this paper, we use an improved array steering vector $\tilde{\mathbf{a}}$, by taking into consideration the array aperture's radiation property [9].

$$
\begin{aligned}
& \tilde{\mathbf{a}}(f, \theta, \varphi, \mathbf{r}) \\
& \quad=\left[g_{1}(f, \theta, \phi), g_{2}(f, \theta, \phi) e^{j k \mathbf{r}_{2}(\theta, \varphi)}, \ldots, g_{M}(f, \theta, \phi) e^{j k \mathbf{r}_{M}-1(\theta, \varphi)}\right],
\end{aligned}
$$

where $g_{i}(f, \theta, \varphi)$ is the known active gain response of the $i$ th antenna. It can be obtained during antenna design using electromagnetic simulation software or through actual measurements.

This new steering vector $\tilde{\mathbf{a}}$ in (4) is a closer approximate to the real steering vector $\widehat{\mathbf{a}}$, compared to the one a without considering mismatches. This can be seen from the simulation results as will be presented in Section 5 .

\section{Proposed APWC Algorithm}

Define the approximation error for the radiation pattern expressions with and without considering mismatches as

$$
\begin{aligned}
& \tilde{\mathbf{e}}=\widehat{\mathbf{a}}-\tilde{\mathbf{a}}, \\
& \mathbf{e}=\widehat{\mathbf{a}}-\mathbf{a},
\end{aligned}
$$

respectively.

Assume that the norm of $\tilde{\mathbf{e}}$ is bounded by a known constant $\tilde{\varepsilon}>0$. We can formulate the APWC problem as a constrained minimization problem

$$
\begin{aligned}
\min _{\mathbf{w}} & \mathbf{w}^{H} \widehat{\mathbf{R}} \mathbf{w}, \\
\text { subject to } & \left|\mathbf{w}^{H} \tilde{\mathbf{c}}\right| \geq 1 \\
& \text { for all } \tilde{\mathbf{c}} \in A(\tilde{\varepsilon}), \\
& A(\tilde{\varepsilon}) \triangleq\{\tilde{\mathbf{c}} \mid \tilde{\mathbf{c}}=\tilde{\mathbf{a}}+\tilde{\mathbf{e}},\|\tilde{\mathbf{e}}\| \leq \tilde{\varepsilon}\} .
\end{aligned}
$$

It can be rewritten as

$$
\begin{array}{ll}
\min _{\mathbf{w}} & \mathbf{w}^{H} \widehat{\mathbf{R}} \mathbf{w}, \\
\text { s.t. } & \left(\mathbf{w}^{H} \tilde{\mathbf{a}}-1\right) /\|\mathbf{w}\| \geq \tilde{\varepsilon}, \\
& \operatorname{Im}\left\{\mathbf{w}^{H} \tilde{\mathbf{a}}\right\}=0 .
\end{array}
$$

The APWC method belongs to the class of DL method. Similar to the WCRB method [2], the weight solution to (7) can be derived to be

$$
\mathbf{w}=\tilde{\beta}(\widehat{\mathbf{R}}+\tilde{\xi} \mathbf{I})^{-1} \tilde{\mathbf{a}}
$$

where $\tilde{\beta}=\lambda /\left[\lambda \mathbf{a}^{H}\left(\widehat{\mathbf{R}}+\lambda \tilde{\varepsilon}^{2} \mathbf{I}\right)^{-1} \mathbf{a}-1\right], \lambda$ is a Lagrange multiplier, and $\tilde{\xi}=\lambda \tilde{\varepsilon}^{2}$. Equation (7) can also be converted to a convex second-order cone problem and finally solved via interior point method. The computational cost of the APWC algorithm is $O\left(M^{3}\right)$ per iteration.

\section{Simulation Results}

We refer to a practical 4-element uniform circular microstrip array shown in Figure 1 as a standard model in our simulation. This array was developed for an anti-interference subsystem in the BeiDou Navigation System. The commercial electromagnetic simulation software HFSS is used for all antenna simulations.

We simulate array mismatches using HFSS and then abstract and store the corresponding AP for each mismatch. Three types of common mismatches are studied, including (a) the element's position mismatches, (b) size errors of the metal working platform, and (c) dielectric parameter errors 


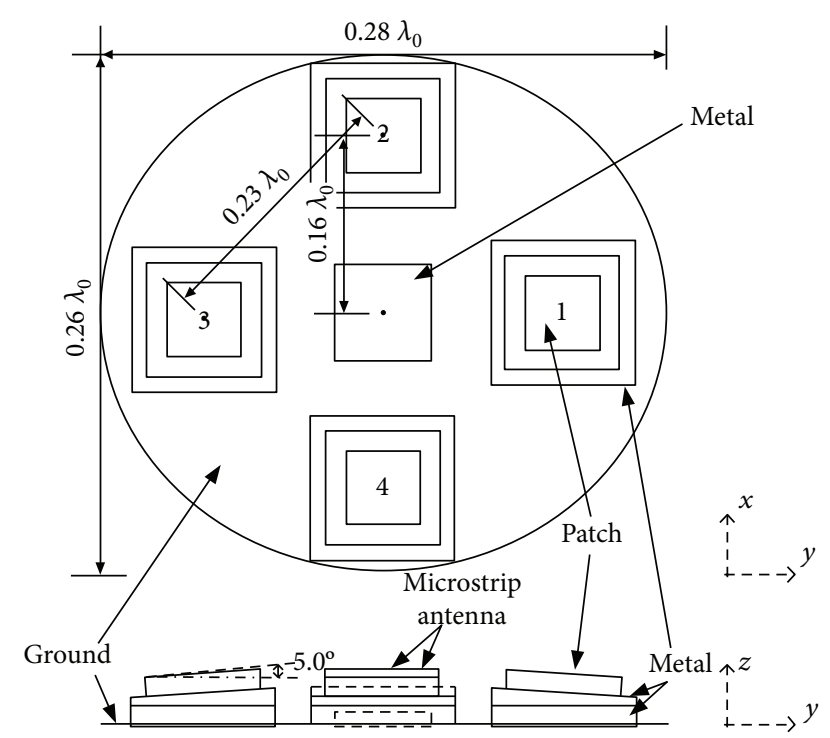

FIGURE 1: A miniature circular microstrip array. The center frequency of each right-hand circular polarized element is $f_{0}=1.268 \mathrm{GHz}$. The dielectric substrate has RDP of $\varepsilon_{r}=20$ and LT of $\tan \delta=0.004$. The metal working platform is elliptical, with a $2 a \approx 0.28 \lambda=134 \mathrm{~mm}$ major axis and a $2 b \approx 0.26 \lambda=121 \mathrm{~mm}$ minor axis. The array's radius is $r=0.16 \lambda=38 \mathrm{~mm}$, and the interelement spacing is $d=0.23 \lambda=54 \mathrm{~mm}$. Each antenna is set at an inclined angle of $\theta=5^{\circ}$ on the workbench.

of elements' substrate, including relative dielectric permittivity (RDP) and loss tangent (LT). The sample covariance matrix $\widehat{\mathbf{R}}$, which reflects the varying electromagnetic environment, varies with different prestored AP data. Using some of the data, Figure 2 compares the norm $\tilde{e}=\|\tilde{\mathbf{e}}\|$ and $e=\|\mathbf{e}\|$ in (5) under different types of mismatches. The results clearly show that our adopted radiation expression with AP in (4) approximates the real radiation pattern much better than the one without considering mismatch.

We assume that the desired signal and interference have a plane wavefront with $\left(\theta_{s}, \varphi_{s}\right)=\left(70^{\circ}, 6^{\circ}\right)$ and $\left(\theta_{i}, \varphi_{i}\right)=\left(1^{\circ}, 90^{\circ}\right)$, respectively. For each simulated point, we ran 200 implementations. The input signal-to-noise ratio (SNR) and interference-to-noise ratio (INR) in a single antenna element equal to $25 \mathrm{~dB}$ and $30 \mathrm{~dB}$, respectively. Signals are in the training data cell (training data size $N=100$ unless stated otherwise). Additive noise in the array is modelled as spatially and temporally independent complex Gaussian noise with zero mean and unit variance. The mean output SINR for the following five algorithms is compared under mismatch situations: the SMI beamformer, the LSMI method [1], the AP beamformer [10], the WCRB method [2], and the APWC method using the SeDuMi convex optimization MATLAB toolbox. The optimal SINR, $\mathrm{SINR}_{\mathrm{opt}}=\sigma_{s}^{2} \mathbf{a}_{\mathrm{s}}{ }^{H} \boldsymbol{R}_{i+n}^{-1} \mathbf{a}_{\mathrm{s}}$, is also shown.

Figure 3 shows how beamformers' output SINR is affected by antenna elements' position mismatch. Figure 4 illustrates how the performance changes with the size of the working platform, as an example of the working environment variation. It indicates that the size variation of the metal

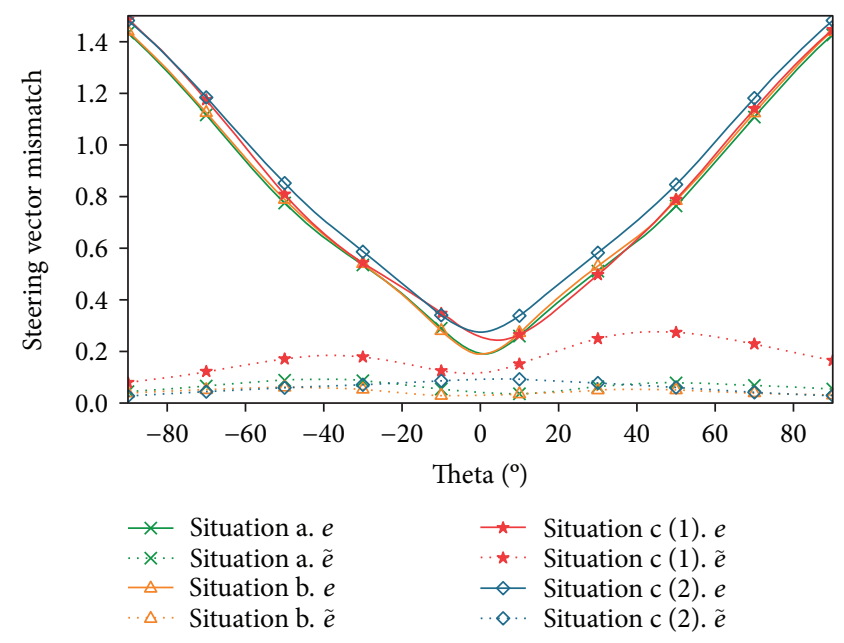

FIGURE 2: Steering vector mismatches (norm of the difference) under different situations: a: element 1's position mismatch $(+2 \mathrm{~mm}$ along axis $y$ ), b: working platform mismatch $(2 a=136 \mathrm{~mm})$, and $\mathrm{c}$ : (1) element 1's RDP mismatch $\left(\varepsilon_{r}=20.3\right)$ and (2) element 1's LT mismatch $(\tan \delta=0.005)$.

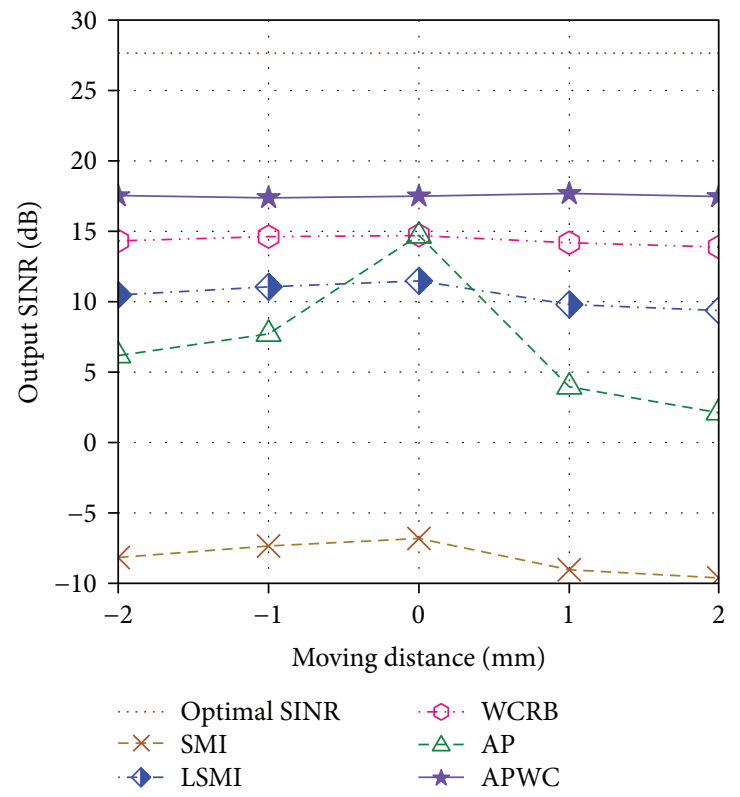

FIgURE 3: Output SINR versus antenna elements' position. Element 1 is moved along the $y$-axis from $-2 \mathrm{~mm}$ to $2 \mathrm{~mm}, 1 \mathrm{~mm}$ at a time.

working platform can cause the change of edge scattering and affect the electromagnetic radiation, particularly for AP and SMI algorithms. Both figures show that the proposed APWC method outperforms other methods with improved SINR and robustness.

In Figures 5 and 6, we present how the output SINR changes with the substrate's RDP and LT (the range of variation is determined by engineering experience). From the two figures, we can see that the proposed APWC method also achieves improved SINR and robustness under these two types of mismatches. 


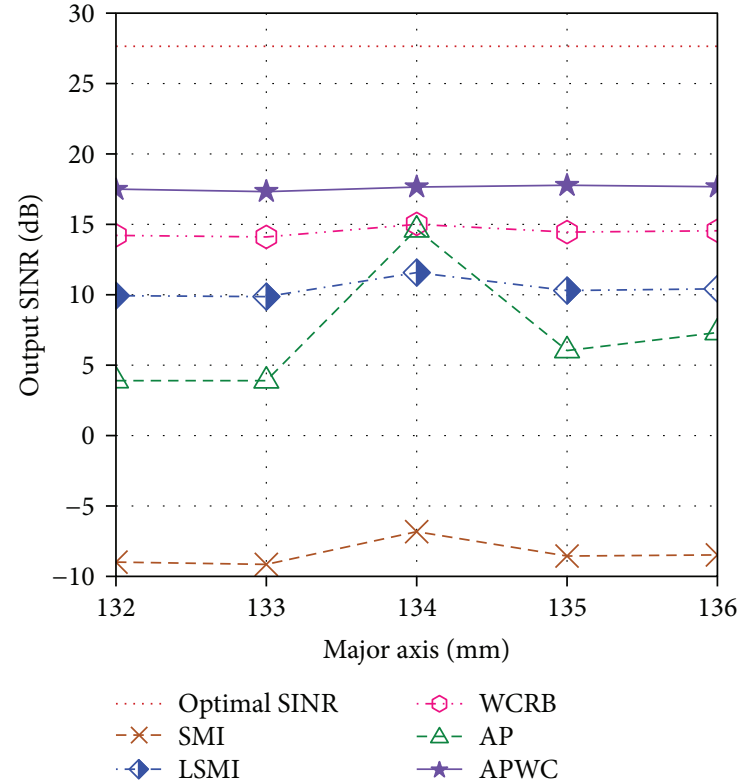

FIgURE 4: Output SINR versus the size of the working platform. The length of the elliptical platform's major axis varies from $132 \mathrm{~mm}$ to $136 \mathrm{~mm}$

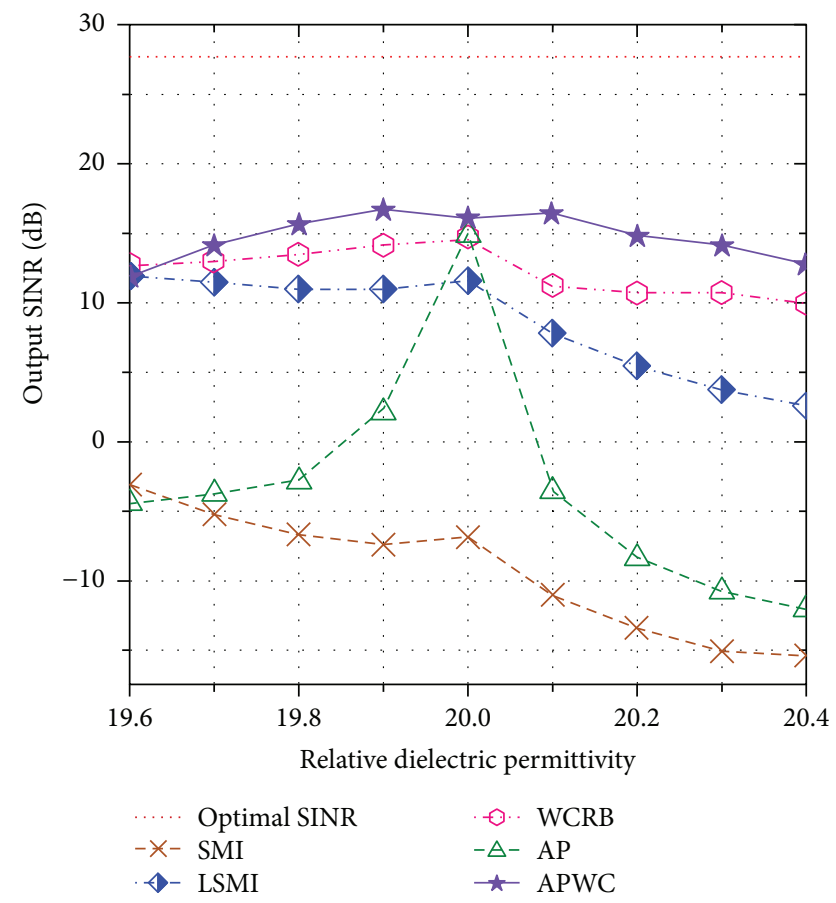

FIGURE 5: Output SINR versus the substrate's RDP. Element 1's RDP varies from 19.6 to 20.4.

\section{Measured Results}

The array mentioned in Section 5 has been fabricated, debugged, and then measured in the microwave anechoic chamber. Due to the small mismatches introduced in the process of fabrication, we can expect a numerical difference

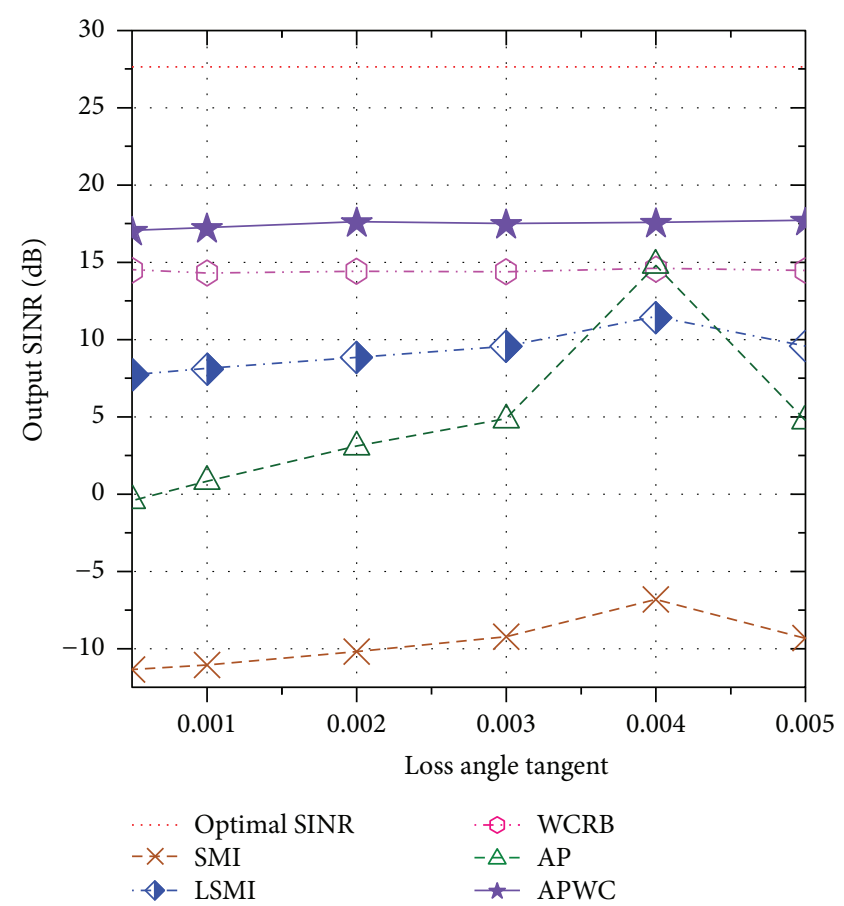

FIgURE 6: Output SINR versus substrates' LT. Each element's LT varies from 0.0005 to 0.005 .

TABLE 1: The active gain of designed array $\left(G_{\mathrm{d}}\right)$ and fabricated array $\left(G_{\mathrm{f}}\right)$ from the DoAs of signal and interference.

\begin{tabular}{lcccc}
\hline \multirow{2}{*}{ Element's number } & \multicolumn{2}{c}{$G_{\mathrm{d}}(\mathrm{dB})$} & \multicolumn{2}{c}{$G_{\mathrm{f}}(\mathrm{dB})$} \\
& $\theta_{s}=70^{\circ}$ & $\theta_{i}=1^{\circ}$ & $\theta_{s}=70^{\circ}$ & $\theta_{i}=1^{\circ}$ \\
& $\varphi_{s}=6^{\circ}$ & $\varphi_{i}=90^{\circ}$ & $\varphi_{s}=6^{\circ}$ & $\varphi_{i}=90^{\circ}$ \\
\hline 1 & -4.91 & -0.40 & -3.03 & -0.48 \\
2 & -2.84 & -0.50 & -2.53 & -0.54 \\
3 & -3.62 & -0.24 & -3.38 & -0.53 \\
4 & -3.56 & -0.50 & -0.81 & -0.12 \\
\hline
\end{tabular}

of the AP between the designed and practical arrays. Such difference is shown in Table 1, which compares the active gain data of array elements at the direction of arrival (DOA) of signal and interference. With the measured data, we assessed the earlier mentioned algorithms' performance using the same parameters (e.g., DOA and INR) with those in V.

Figure 7 compares the mean SINR for the six methods with a varying number of training snapshots. Figure 8 shows how the mean output SINR changes with different SNRs. Figures 7 and 8 demonstrate that the proposed APWC method consistently achieves better performance than other methods at varying input SNR values and snapshot numbers. We can also compare the simulation and measured results here, referring to the parameters $\mathrm{SNR}=25 \mathrm{~dB}$ and $N=100$ that are available in both results. Compared to the simulation results that are impacted by a single type of mismatch, the SINR of the measured results is reduced by about $2 \mathrm{~dB}$, which is the consequence of combined mismatches. 


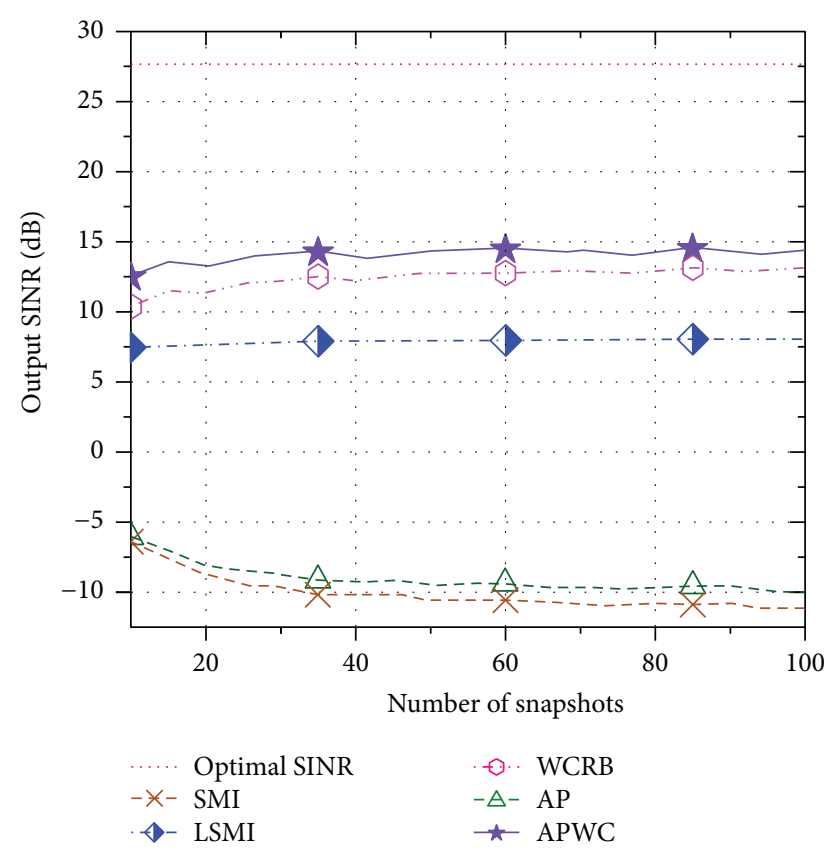

Figure 7: Output SINR versus training data size for $\mathrm{SNR}=25 \mathrm{~dB}$.

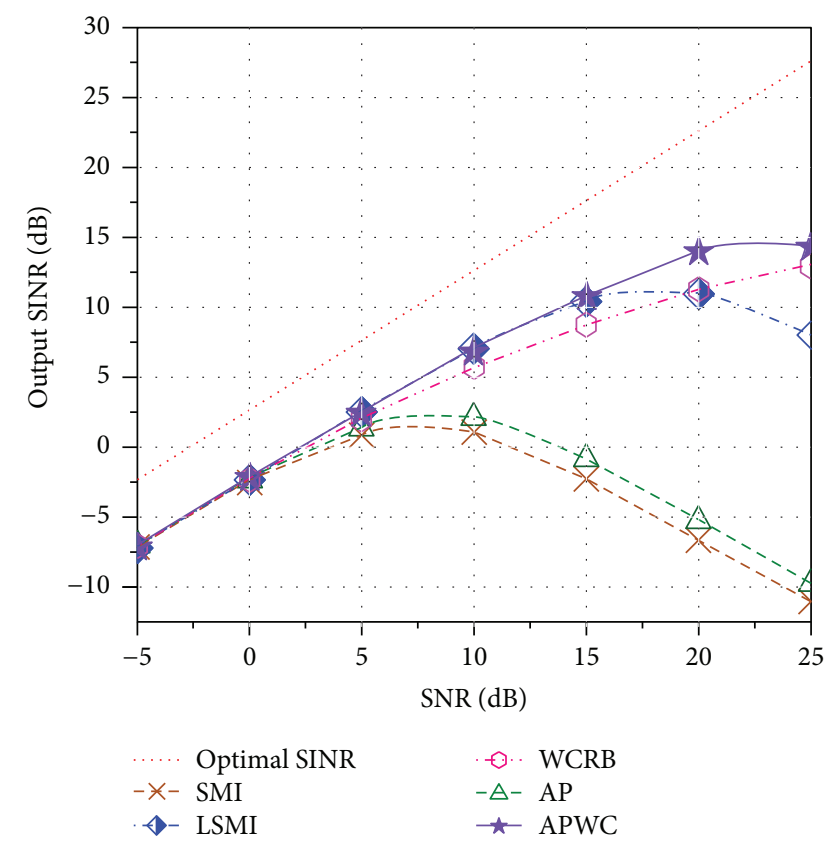

FIGURE 8: Output SINR versus SNR for training data size of $N=100$.

\section{Conclusion}

In this paper, we propose an improved adaptive beamforming algorithm APWC, which embeds the array's electromagnetic characteristics in a robust beamformer. Mathematical analysis, computer simulation, and measured results illustrate the effectiveness and robustness of the proposed algorithm to array manifold mismatches. APWC is particularly suitable for systems with small and compact arrays, where serious mutual coupling and environment scattering could significantly influence antennas' radiation and the performance of conventional beamforming algorithms.

\section{Conflicts of Interest}

The authors declare that they have no conflicts of interest.

\section{References}

[1] H. Cox, R. Zeskind, and M. Owen, "Robust adaptive beamforming," IEEE Transactions on Acoustics, Speech, and Signal Processing, vol. 35, no. 10, pp. 1365-1376, 1987.

[2] S. A. Vorobyov, A. B. Gershman, and Z.-Q. Luo, "Robust adaptive beamforming using worst-case performance optimization: a solution to the signal mismatch problem," IEEE Transactions on Signal Processing, vol. 51, no. 2, pp. 313324, 2003.

[3] J. Li and P. Stoica, Robust Adaptive Beamforming, John Wiley \& Sons, 2005.

[4] A. Khabbazibasmenj, S. A. Vorobyov, and A. Hassanien, "Robust adaptive beamforming based on steering vector estimation with as little as possible prior information," IEEE Transactions on Signal Processing, vol. 60, no. 6, pp. 29742987, 2012.

[5] J. Zhuang, B. Shi, X. Zuo, and A. H. Ali, "Robust adaptive beamforming with minimum sensitivity to correlated random errors," Signal Processing, vol. 131, pp. 92-98, 2017.

[6] J. Xu, G. Liao, L. Huang, and H.-C. So, "Robust adaptive beamforming for fast-moving target detection with FDASTAP radar," IEEE Transactions on Signal Processing, vol. 65, no. 4, pp. 973-984, 2017.

[7] B. Liao, C. Guo, L. Huang, Q. Li, and H. C. So, "Robust adaptive beamforming with precise main beam control," IEEE Transactions on Aerospace and Electronic Systems, vol. 53, no. 1, pp. 345-356, 2017.

[8] V. Joroughi, M. Vazquez, and A. Perez-Neira, "Precoding in multigateway multibeam satellite systems," IEEE Transactions on Wireless Communications, vol. 15, no. 7, pp. 1-4956, 2016.

[9] D. F. Kelley and W. L. Stutzman, "Array antenna pattern modeling methods that include mutual coupling effects," IEEE Transactions on Antennas and Propagation, vol. 41, no. 12, pp. 1625-1632, 1993.

[10] R. Goossens and H. Rogier, "Optimal beam forming in the presence of mutual coupling," in 2006 Symposium on Communications and Vehicular Technology, pp. 13-18, Liege, Belgium, 2006.

[11] B. P. Ng, "Array shape self-calibration technique for direction finding problems," IEE Proceedings H Microwaves, Antennas and Propagation, vol. 139, no. 6, p. 521, 1992.

[12] S. Henault and Y. Antar, "Unifying the theory of mutual coupling compensation in antenna arrays," IEEE Antennas and Propagation Magazine, vol. 57, no. 2, pp. 104-122, 2015.

[13] J. Ruze, “Antenna tolerance theory-a review," Proceedings of the IEEE, vol. 54, no. 4, pp. 633-640, 1966. 


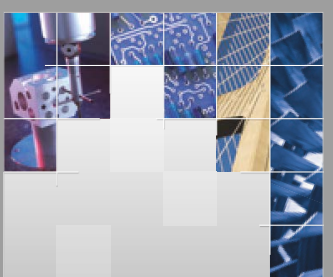

\section{Enfincering}
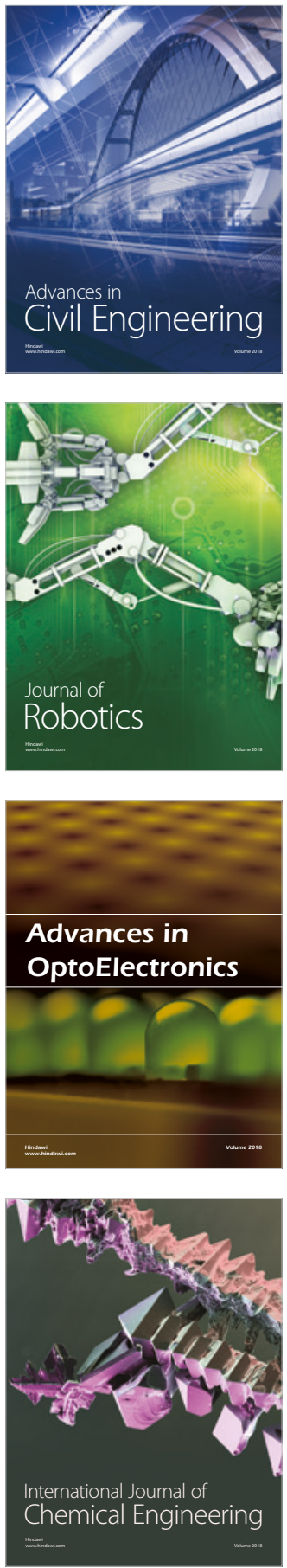

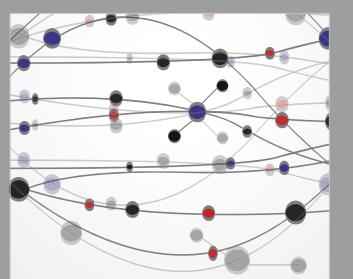

\section{Rotating \\ Machinery}

The Scientific World Journal

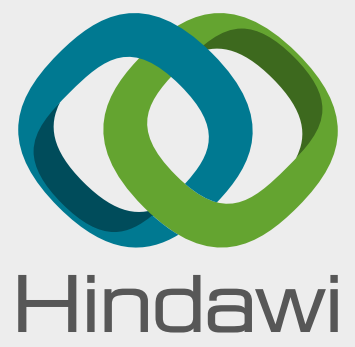

Submit your manuscripts at

www.hindawi.com
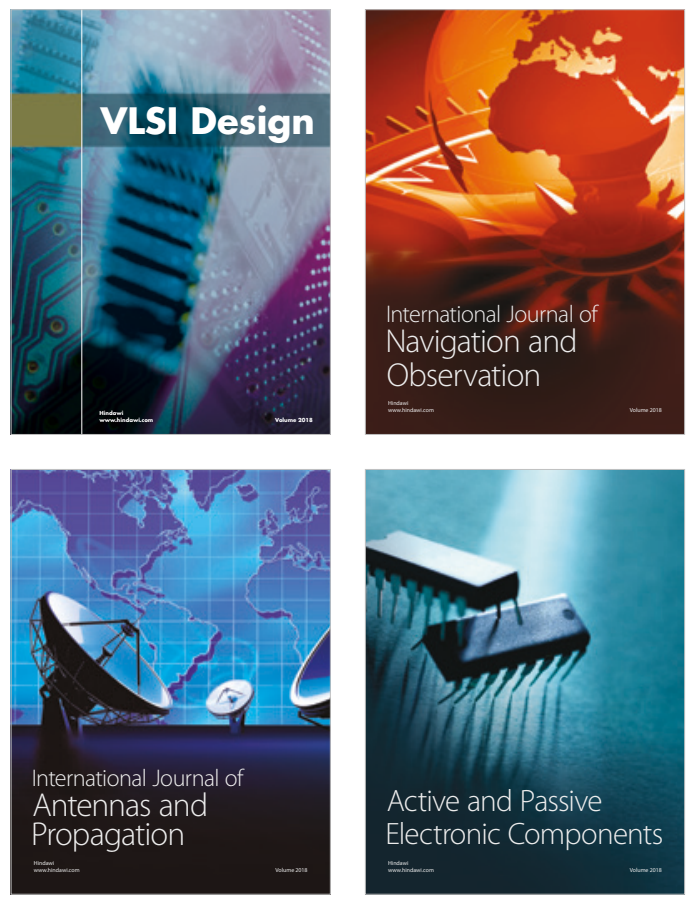
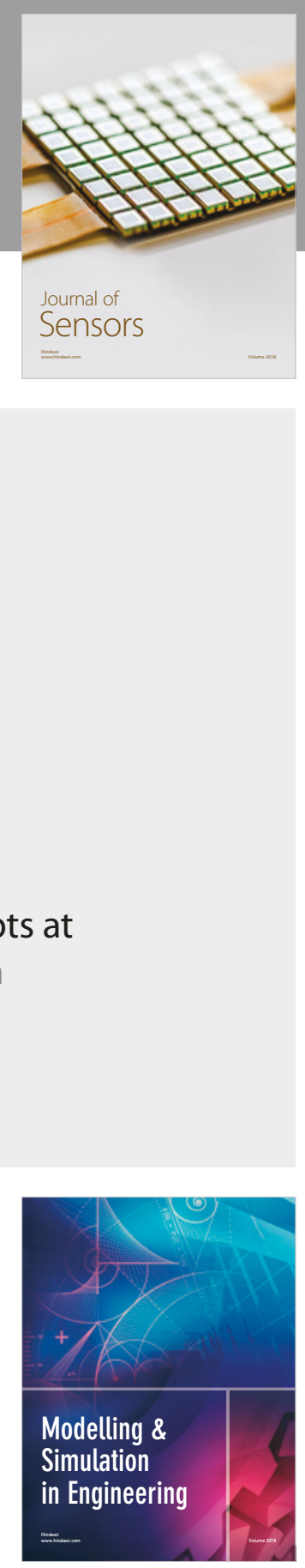

\section{Advances \\ Multimedia}
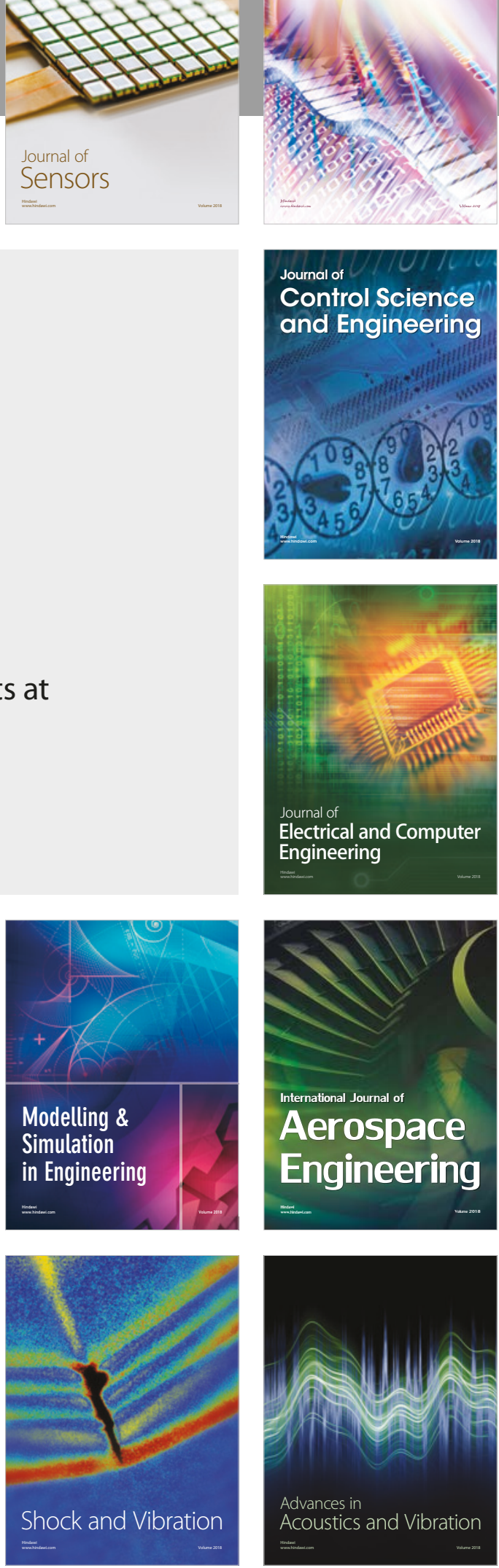